\title{
Verb types and semantic maps
}

\author{
Marloes Oomen (ACLC, University of Amsterdam) \\ m.oomen2@uva.nl
}

\begin{abstract}
Verb classifications for sign languages are typically based on agreement properties (Padden 1988) and have also been suggested to be semantically grounded (Meir 1998, 2002). In light of the latter proposition, it is surprising that the semantics of verb types have not been explored in much depth. This study aims to investigate the semantic underpinnings of verb types. It is hypothesized that the semantic properties that govern case-frame selection in spoken languages (Hopper and Thompson 1980; Tsunoda 1981) similarly mediate verb type in sign languages. The rationale behind this idea is that many such properties have the potential to be expressed iconically in sign language verb forms. Particular constellations of features are thus expected to increase the likelihood of a verb to be of a certain type. To test this hypothesis, a method previously used to investigate the connection between verb semantics and transitivity in spoken languages (Malchukov 2005) is applied to data from German Sign Language (DGS). Overall, the results lend credibility to the supposition that case marking systems in spoken languages and the verb-type system in sign languages are sensitive to the same underlying semantic factors, underscoring the centrality of these notions in language. In addition, the results offer a finer-grained picture of the semantics of verbs of different types, and enable us to formulate predictions about the scope of diachronic change and synchronic and cross-linguistic variation in this domain.
\end{abstract}

Keywords: verb classification, semantic map, transitivity, German Sign Language

\section{Introduction}

Verbs in sign languages are commonly classified based on their agreement properties. Typically, a distinction is made between agreement verbs and spatial verbs - which agree with person/location - and plain verbs - which do not agree (see e.g. Padden 1988; Janis 1992; de Quadros 1999; Meir 2002). It has also been suggested that this classification is semantically grounded: agreement verbs have been said to denote transfer, spatial verbs motion, and plain verbs neither of the two (Meir 1998, 2002). Given the typologically singular proposition that the verb agreement system is rooted in semantics in sign languages, it is perhaps surprising that details of the semantics of different verb types have not been explored in much more depth beyond what Meir $(1998,2002)$ has offered. The aim of this paper, therefore, is to gain a deeper understanding of the semantic underpinnings of verb types.

I start from the intuition that there is an intricate connection between transitivity and sign language verb type which is governed by properties of events and their participants. The central hypothesis is that verb semantics impacts on sign language verb type quite like the way in which it affects case-marking for transitivity in spoken languages (following Hopper \& Thompson, 1980 and Tsunoda, 1981, among others). If there is any truth to this hypothesis, 
then it should be possible to apply methods that have been used to investigate transitivity in spoken languages to sign language data. This is what I set out to endeavor.

I outline my approach in Section 2 and introduce the method I will apply to data from one particular sign language - German Sign Language (DGS) - in Section 3. In Section 4, I discuss the DGS corpus data used in this study; Section 5 reports on the results. The exercise leads to a number of generalizations and predictions about verb types across sign languages (Section 6). Section 7 concludes the paper.

\section{Verb semantics, verb types, and transitivity}

I entertain the idea that the realization of sign language verbs as particular types is mediated by the same semantic properties that influence the selection of case as an indicator of the transitivity of verbs in spoken languages. A fundamental insight leading into this idea is that - by default - prototypical agreeing verbs in sign languages denote transitive events involving (at least) two participants, as they have the ability to agree with two arguments. Indeed, Meir $(1998,2002)$ claims that agreement verbs express transfer - a statement that aligns rather well with the traditional view of prototypical transitivity as "a matter of [...] transferring an action from one participant to another” (Hopper and Thompson 1980:253).

It is not a coincidence, of course, that agreement verbs tend to denote concepts involving transfer: the path movement that most agreement verbs possess iconically represents such a relation. But a transfer relation is not the only semantic feature that can be expressed in an iconic manner; the visuo-spatial nature of the signed modality creates a vast array of possibilities in this regard. I thus hypothesize that particular constellations of iconically motivated properties increase the likelihood of a verb to be of a certain type. To give a concrete example, the verb meaning fear denotes a psychological state, of which one of the main hallmarks is that it involves (at least) an experiencer. As pointed out previously by Meir et al. (2007) and Oomen (2017), body-anchoring causes the signer's body to iconically represent such an experiencer. Indeed, each of the three DGS verb forms denoting fear attested in the corpus data (see Section 4) is body-anchored.

But there are other properties of verb meanings that may be conveyed in an iconic manner. For instance, fear is caused by a certain stimulus or trigger. In the three body-anchored forms in DGS, this aspect of the verb's meaning is not represented through an iconic formto-meaning mapping. However, it is not unthinkable that a form would make reference to this event participant. Indeed, there are sign languages with psych-verbs that agree with two arguments through directionality. The verb HATE in ISL is of this type (Meir 1998): it has a path movement from experiencer to stimulus. While we may expect both intra- and crosslinguistic variation with respect to which aspects of events are highlighted in individual verb forms, the premise on which this study is built is that the variation is not random.

Significantly, properties of events and their participants are known to govern case-frame selection as an indicator of transitivity in spoken languages. I define transitivity as a gradable and semantically multifactorial notion following Hopper and Thompson (1980). That is, verbs denoting concepts that involve many properties associated with a high degree of transitivity are more likely to occur in a transitive (e.g. nominative/accusative) case-frame than verbs that with fewer such properties. If the hypothesis that the same sort of properties that govern verb type in sign languages also mediate transitivity marking in spoken languages has any bearing, then it should be possible to apply a semantic map for transitivity splits 
(Malchukov, 2005; Section 3) to the DGS data. ${ }^{1}$

Semantic maps makes predictions about the multifunctionality of grammatical elements. In this chapter, I treat different verb types as distinct grammatical elements, too. More precisely, the directionality of agreeing verbs, the body-anchoring of body-anchored verbs, and the localizing ability of neutral verbs are treated as grammatical markers. If verb type and transitivity marking are indeed governed by the same semantic properties, then the predictions Malchukov's (2005) semantic map makes should, in principle, not be violated.

Regardless of whether or not the DGS data obey the restrictions the map imposes, using this tool to classify DGS verb forms makes it possible to evaluate, with a fair degree of sophistication, the semantic profiles of different verb types in DGS, and - given the similarities across sign languages in this domain - sign languages in general.

\section{A semantic map}

A semantic map is a typological tool used to represent the multifunctionality of grammatical elements in a network (Haspelmath 2003). Semantic maps are intended to broaden our understanding of structural - and potentially universal - semantic patterns by mapping out semantic categories in such a way that multifunctional linguistic elements occupy contiguous spaces on the map. Very often, different functions of grammatical elements are related; a semantic map is a way to chart such functions with the aim of making cross-linguistic predictions about their scope. ${ }^{2}$ An advantage of a semantic map is that it does not presuppose prototypical functions for grammatical markers. The method also facilitates the process of drawing cross-linguistically valid conclusions about a wide variety of linguistic phenomena - even and especially in particularly complex domains with large amounts of cross-linguistic variation.

When constructing a semantic map for a particular domain, a sample of typologically diverse languages has to be studied to identify functions that can be expressed by the same grammatical markers, and vice versa. The functions then need to be arranged in a semantic map such that for each language, every marker covers a contiguous area on the map (Haspelmath 2003). As such, a semantic map expresses implicational universals: if a particular marker in a language expresses function $\mathrm{A}$ and function $\mathrm{C}$, it should also express intervening function B.

Inspired by the pioneering work by Hopper and Thompson (1980), and further building on work by Tsunoda (1981) on transitivity splits of lexical verbs, Malchukov (2005) introduces a semantic map that makes predictions about the scope of transitivity marking across languages (Figure 1). The map introduces semantic categories as functions and predicts that if some - not all - members of two semantic categories take a certain case-frame, then at least some members of any intervening categories should do so too.

The map in Figure 1 integrates three dimensions of transitivity. The upper strand ('effective action' - 'contact' - 'pursuit' - 'motion' - 'intransitive') orders verb classes according to decreasing levels of patienthood of the $\mathrm{O}$ argument (for 'Object', following Dixon 1979).

\footnotetext{
${ }^{1}$ Note that I do not intend to claim that the properties of the different sign language verb types are a form of case marking - although such an analysis is a possible way to go, and one that plays a part in Meir's (1998) analysis of agreement verbs.

${ }^{2}$ Whether the functions of grammatical elements are described as conventionalized meanings or contextually determined uses depends on one's theoretical assumptions. Following Haspelmath, I simply use the term 'function' here. Similarities in function are often rooted in semantics, but not always; functions can also be differentiated pragmatically (Hengeveld and Lier 2010) or syntactically (Haspelmath 1999), for instance.
} 
The middle dimension of transitivity concerns referential distinctness (following Kemmer 1993). The categories 'reflexive', 'middle', and 'spontaneous' include syntactic reflexives, inherent (semantic) reflexives and inherent reciprocals, and verbs of spontaneous action, respectively. The lowest pathway sorts verb classes according to decreasing agentivity of the A argument (for 'Agent', following Dixon 1979) as well as decreasing affectedness of the O argument. In other words, A becomes increasingly more experiencer-like, while $\mathrm{O}$ becomes increasingly more like a stimulus or causer. The class of interaction verbs is sandwiched in between the upper and middle strand. The category 'intransitive' on the far right of the map is not discussed in detail by Malchukov but seems to serve as a repository for stative, nonagentive intransitive verbs that cannot be categorized elsewhere. Note that the category is thus defined in grammatical rather than semantic terms. The connecting lines between categories indicate (semantic) closeness of the classes and represent hypotheses about the scope of transitivity frames as well as pathways of grammatical change. All DGS verb forms that were annotated according to the procedure outlined in the next section will be classified into one of the categories on the map in Figure 1 (see Section 5).

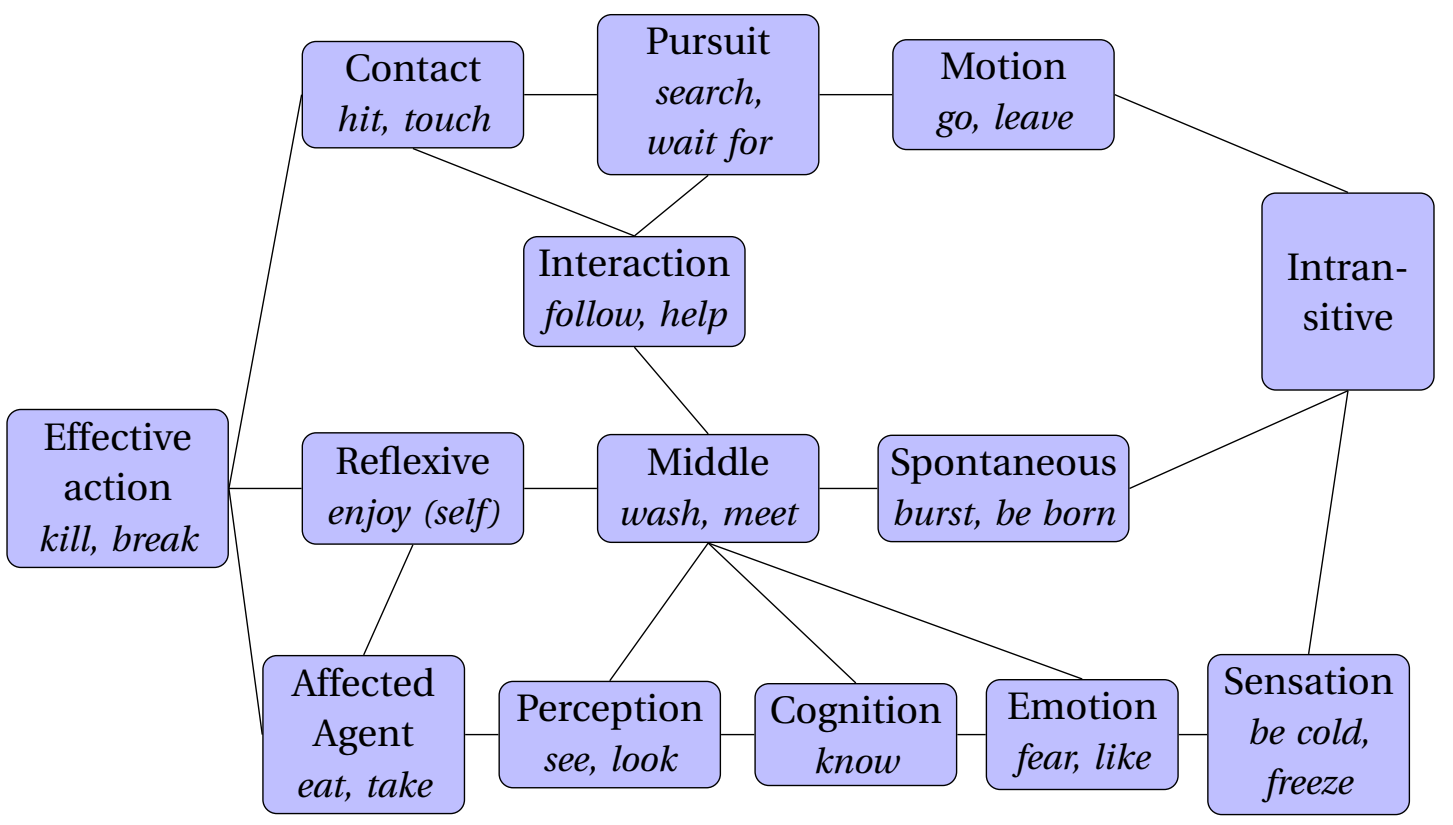

Figure 1: Semantic map for transitivity splits (Malchukov 2005) with examples for each category in italics. Malchukov (2005) does not provide any examples for the category 'Intransitive'.

\section{Annotation of the DGS data}

A total of 58 glossed and translated dialogues from the DGS Corpus (Blanck et al. 2010) - approximately 8.5 hours of material - form the basis for this study. Annotations were added in ELAN (Sloetjes and Wittenburg 2008) to all clauses in the data set that include lexical verbs denoting any of the 80 verb meanings in the 'ValPaL' list (Hartmann, Haspelmath, and Taylor 2013), which was created by a team of typologists to facilitate typological work on valency patterns across languages. The verb meanings included in the list are intended to be representative of the verbal lexicon and are known to display distinctive syntactic behavior, both intra- and cross-linguistically. 
Often, more than one lexical form denoting the same meaning was attested; in such cases, they are distinguished by means of number suffixes. Synonyms, antonyms, or otherwise closely related signs that can reasonably be expected to display identical morphosyntactic behavior (e.g. DRINK in addition to EAT) were also annotated. For each token, verb type was indicated on a separate tier. ${ }^{3}$ I distinguish three types of verbs; note that they do not fully correspond to Padden's (1988) classification. The class of agreeing verbs includes both agreement and spatial verbs, following previous suggestions in the literature that these categories should be collapsed (Janis 1992; de Quadros 1999). I distinguish an additional two types which have traditionally been grouped together in Padden's (1988) category of plain verbs. Verbs that are articulated on the body are classified as body-anchored verbs following suggestions that such verbs have distinct morphosyntactic properties (Meir et al. 2007; Oomen 2017). Finally, verbs articulated in neutral space that do not agree through directionality although at least some of them may agree with a single argument through localization - were annotated as neutral verbs. Verb type was determined for each verb form on the basis of the articulatory properties mentioned. Since many tokens (1560 in total) were annotated, it was almost always possible to determine categorization on the basis of more than just one token per verb form. As will be described in Section 5.4, this was particularly useful in the case of 'hybrid' verb forms with properties of more than one type.

\section{DGS verbs on the semantic map}

All of the DGS verb forms identified in the corpus data were sorted into one of the semantic classes on Malchukov's (2005) semantic map (Figure 1). In addition to Malchukov (2005), two other sources were consulted for this process. ${ }^{4}$ Tsunoda (1981) was used because Malchukov's map is an adaptation and extension of Tsunoda's transitivity hierarchy, and Levin (1993) was consulted because Malchukov mentions her classification of English verbs as a source for his semantic classification. Here are several examples of how lexical forms were classified:

- BREAK is categorized as a verb of effective action based on Malchukov (2005).

- SMELL1 and SMELL2 are categorized as perception verbs based on Tsunoda (1981); Malchukov does not mention this verb meaning.

- BRING is categorized as an affected Agent verb because Levin (1993) groups bring and take together in a two-member class, and Malchukov describes take as an affected Agent verb.

- HUG1 and HUG2 are categorized as both interaction and middle verbs (and thus positioned on the map between these two categories): according to Levin (1993), hug belongs to the same verb category as marry, which Malchukov positions between the 'interaction' and 'middle' categories.

\footnotetext{
${ }^{3}$ Since the present study is part of a larger project on argument structure in sign languages, many more annotations (e.g. for word order and agreement properties) were added for each token. None of these annotations are relevant for the current study.

${ }^{4}$ I deliberately avoided making my own judgments about semantic-class membership to escape the risk of circularity, i.e. sorting a verb into a semantic category because its agreement properties express certain event properties that render a particular semantic interpretation more likely.
} 
The full map is displayed in Figure 3. The different shades of blue of the verb labels indicate verb type. Body-anchored verbs are the lightest blue, followed by neutral verbs and agreeing verbs in increasingly darker blue tones. 'Weather verbs' are added as a separate category connected to the class of 'intransitives', which I use to include verbs that are classified by Levin (1993) as verbs of existence. The categories 'reflexive' and 'middle' distinguished by Malchukov (2005) are grouped together in Figure 3 because a distinction between members of the two categories can only be made on syntactic grounds, while the aim here is to categorize all of the lexical forms on the basis of the semantics of the corresponding verb meanings. In the Appendix to this paper, the reader can find individual maps for each of the verb types for convenience (Figure 6-8). The map for agreeing verbs (Figure 8) additionally distinguishes prototypical agreeing verbs from spatial verbs. In the next sections, each verb type is discussed in turn.

\subsection{Body-anchored verbs}

According to prediction, the categories on the map that contain body-anchored verbs are contiguous (see Figure 6 in the Appendix for a clearer picture) - although it should be noted that almost every semantic category on the map contains at least one verb of this type. Still, the largest concentration of body-anchored verbs can clearly be found in the categories on the bottom strand of the map, which orders semantic classes according to decreased agentivity of A correlated with decreased affectedness of $O$. The observed pattern is hardly surprising: body-anchoring commonly serves to make iconic reference to a referent's internal experience or the expression thereof (Meir et al. 2007; Oomen 2017), thus emphasizing less agentive properties of the A argument. ${ }^{5}$ FEAR 1 (Figure $2 \mathrm{a}$ ) is an example of such a form.

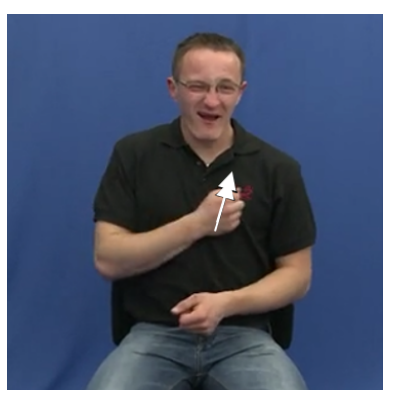

(a) FEAR 1

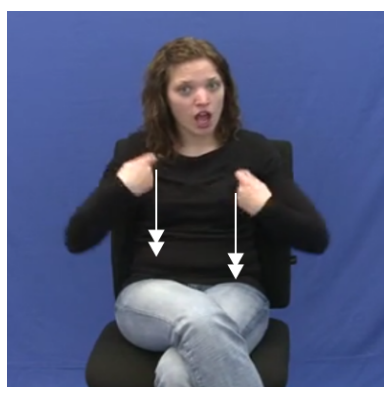

(b) DRESS 1

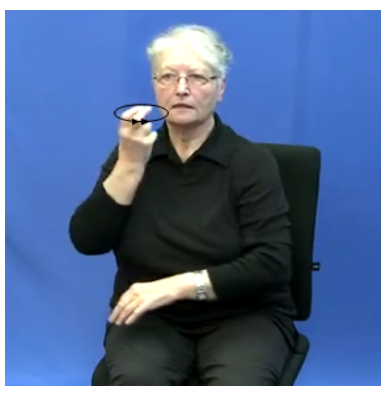

(c) SEARCH-FOR 1

Figure 2: Three body-anchored verbs.

Body-anchored verb forms are also fairly common in the 'reflexive/middle' and 'interaction' categories. Again, the reasons are obvious. Lexical reflexives, such as DRESS 1 (Figure 2b), denote actions that one performs on oneself and therefore typically involve the body. The class of interaction verbs includes several verbs of speech or saying, with the corresponding lexical forms all making reference to the mouth.

But body-anchored verbs are also included, albeit more sparsely, in the categories 'pursuit', 'motion', 'spontaneous', and 'intransitives'. To give an example, the pursuit verb SEARCH FOR 1, depicted in Figure 2c, is body-anchored because its place of articulation near the face is iconically motivated; it refers to an entity performing a search using their eyes. The verb form emphasizes the action performed by the searcher. RUN 1 and RUN2 refer to the way people move their arms when running. No reference is made to the trajectory of a run, which

\footnotetext{
${ }^{5}$ The stimulus/causer, on the other hand, is backgrounded in such sign language verb forms.
} 


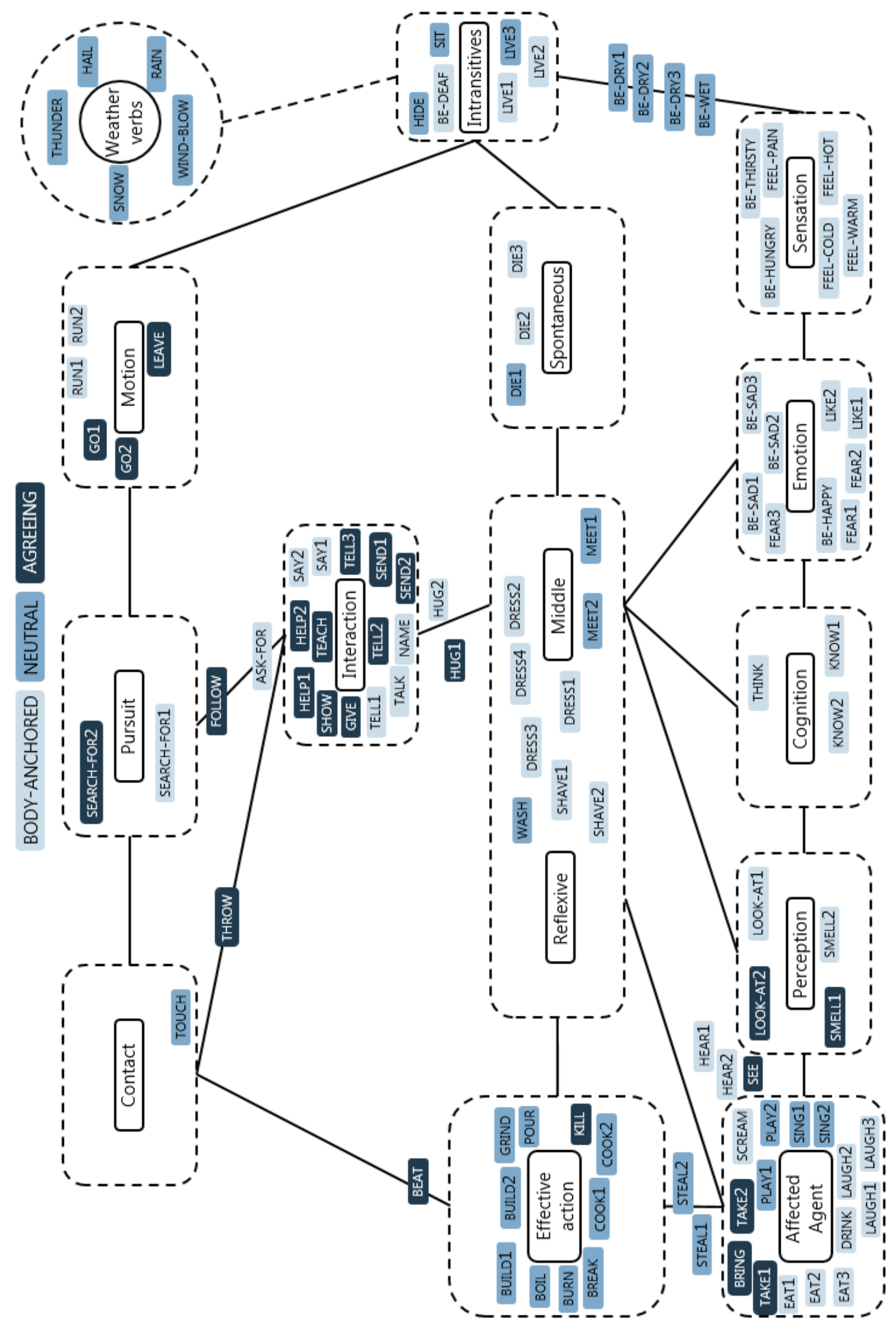

Figure 3: The semantic map for transitivity splits (Malchukov 2005) with the DGS data. 
can only be expressed with a classifier predicate. Thus, the body-anchored verbs in these categories seem to emphasize different aspects of the events they denote than one might perhaps expect.

\subsection{Neutral verbs}

For neutral verbs (Figure 7), we can observe a clearly discernible cluster of verb forms on the left side of the map: approximately half of all neutral verb forms are located in or between the 'effective action' and 'affected Agent' categories. At the opposite end of the map, we also find a fair number of verb forms in or around the category of intransitives. Connecting these two poles - and ensuring that all the categories with neutral verbs are contiguous - are a handful of forms in the 'reflexive/middle' and 'spontaneous' categories.

Three examples of neutral verb forms are given in Figure 4. COOK1, an effective action verb, involves a manual articulation that references a person cooking (or, more literally, stirring), while its place of articulation - which may be modified - refers to the stuff being cooked. SIT (Figure 4b), classified as 'intransitive', makes reference to just a single argument, the sitter, by means of both the handshape and (optional) localization. The two verb forms denoting MEET (MEET 1 is depicted in Figure 4c) reference reciprocity by means of the converging movement of the hands, which each represent individuals.

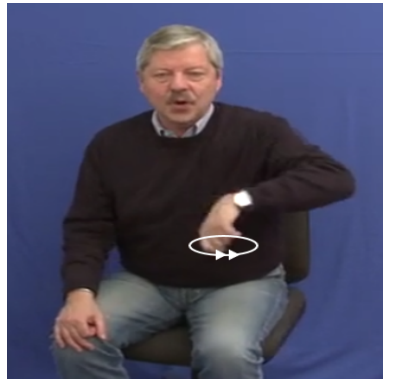

(a) COOK 1

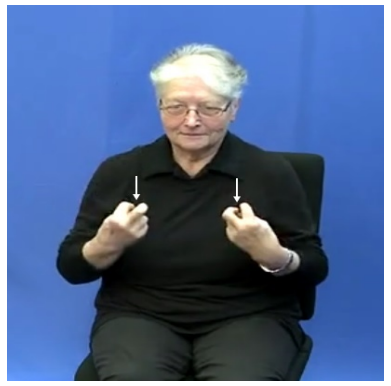

(b) SIT

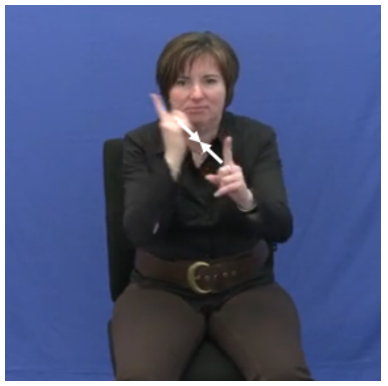

(c) MEET 1

Figure 4: Three neutral verbs.

The semantic profile of neutral verbs is radically different from that of body-anchored verbs: there are hardly any neutral forms in the categories on the lower dimension, while bodyanchored verbs do not feature at all in the most prototypical semantic category for neutral verbs, namely that of effective action. As such, the maps provide a semantic argument for distinguishing between verbs of the two types.

\subsection{Agreeing verbs}

Agreeing verbs (Figure 8) cluster around categories on the upper dimension of the map, which orders categories according to decreased affectedness of the $\mathrm{O}$ argument, and toward the left side of the map (i.e. highly transitive verbs). As announced, Figure 8 additionally distinguishes between regular agreeing verbs and spatial verbs. The map reveals that the 'motion', 'pursuit', and 'affected Agent' categories each contain only agreeing verbs of the spatial kind, such as LEAVE (Figure 5c). Regular agreeing verbs, such as TEACH (Figure 5b), are most frequent in the 'interaction' category. A number of forms occur in categories toward the left of the map, such as BEAT (Figure 5a). It is interesting to observe that the map would 
look significantly more fragmented if the class of agreeing verbs were divided into agreement verbs and spatial verbs.

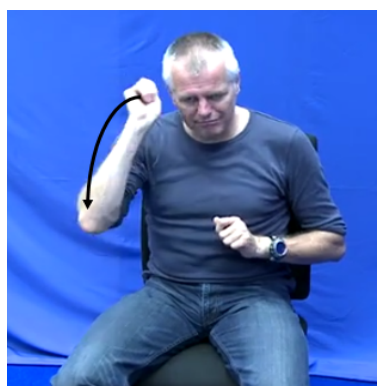

(a) BEAT

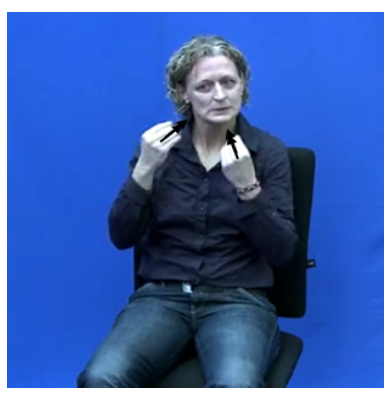

(b) TEACH

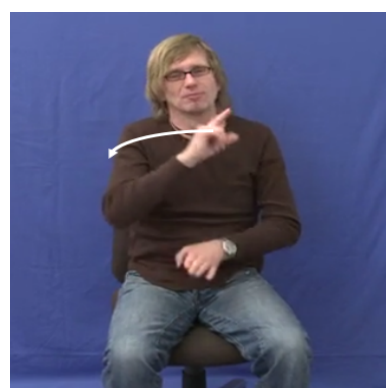

(c) LEAVE

Figure 5: Three agreeing verbs.

It should be noted that the categories 'contact' and 'effective action', which connect the upper strand of the map with the lower strand, only include or share with other categories a handful of lexical forms, while the semantic map predicts that there should be agreeing verbs of these semantic types. It is, of course, possible that this state-of-affairs is simply symptomatic of the limited set of verb meanings that the ValPaL list includes - only few of the verb meanings in the list can be classified as verbs of contact, for instance - but no definitive conclusions can be drawn on the basis of this data set.

Given that both transitive verbs in spoken languages and agreeing verbs in sign languages (Meir 1998, 2002) have been described as verbs of transfer, one might hypothesize that agreeing verbs cluster around the category of 'effective action'; the most transitive category on the map. However, the map in Figure 8 does not provide convincing evidence for this hypothesis. Agreeing verbs are not represented in the left-most categories on the map in high numbers, but concentrate more densely in other areas. It appears that neutral verbs represent the most prototypically transitive concepts. A more accurate general semantic characterization of agreeing verbs may be that they express some form of interaction between participants.

\subsection{Hybrid forms}

Interestingly, some verbs appear to possess characteristics of more than one verb type. SEE, for instance, is articulated with a V-handshape, palm oriented toward the signer's face, and involves a path movement away from the signer. While the verb's trajectory can be modified to agree with the object, the corpus data suggest that the locus at which the movement starts is fixed: it is always in front of the signer's face. As such, SEE has properties of both bodyanchored and agreeing verbs. HUG1 can also express only object agreement. Unlike SEE, it is a backward agreeing verb: the verb starts out at the object locus, and ends at a fixed locus in front of the signer's chest.

A different kind of hybrid is exemplified by the verb MEET1 (illustrated earlier in Figure 4c). This form usually involves a short simultaneous movement of both hands on the midsaggital plane, with the hands converging to make contact approximately at the center of the neutral space. However, in a couple of cases, the starting loci of the two hands are clearly different, suggesting that agreement is expressed with the two (symmetrical) arguments. Thus, MEET 1 appears to be a verb with properties of neutral and agreeing verbs.

Interestingly, each of these verbs forms belong to semantic categories on the map that are at the junction of the domains of the different verb types. MEET 1 even fills up a hiatus in 
the domain of agreeing verbs: verbs that agree are present in categories above, below, as well as to the left of 'reflexive/middle' category in which MEET 1 is placed (see Figure 8), but not in this category itself. Other examples of hybrid verb forms in the corpus data are similarly positioned in overlap spaces. I discuss the potential significance of this observation in the next section.

\section{Discussion}

As noted at the beginning of Section 1, it has previously been argued that verb-type membership is determined, at least to some extent, by verb semantics (Meir 1998, 2002). Yet it is also clear that this is not a rigid correlation. It is known, for instance, that verbs denoting the same meaning in different sign languages may differ with respect to agreement properties - and thus belong to distinct categories. The sign HATE can agree in Israeli Sign Language (ISL; Meir 1998), but it is a body-anchored verb in Sign Language of the Netherlands(NGT; Oomen 2017). UNDERSTAND is a body-anchored verb in many sign languages, including American Sign Language, DGS, and NGT, but it is a (backward) agreement verb in Catalan Sign Language (de Quadros \& Quer 2008). Moreover, verbal signs may change type over time. Meir et al. (2007) and Meir (2012; 2016), for instance, report a diachronic change in the morphosyntactic realization of verbs of transfer in ISL. Over the course of a couple of generations, such verbs are reported to have gradually changed from expressing no agreement, to expressing agreement with the object argument, to expressing agreement with both the subject and the object argument. The findings echo earlier observations by Engberg-Pedersen (1993) for Danish Sign Language. The change always applies to verbs that are originally body-anchored. It seems plausible that some of the hybrid forms I described in Section 5.4 represent intermediate stages in diachronic development.

All of these observations accentuate both the fluidity and the regularity of the sign language verb type system. On the one hand, there are many verbs that may not convincingly fit the mold of any of the categories both within and across sign languages and in either semantic or grammatical terms, or both. On the other hand, it is clear that there are remarkable consistencies across and within sign languages with respect to verb type membership and as far as we can tell - pathways of change.

In light of this discussion, the value of the semantic map with DGS verb forms (Section 5) becomes evident. Since no prototypicality of function is assumed, the map can offer a much more fine-grained picture of verb type semantics, while simultaneously allowing for the plasticity the system presents. For instance, the map can unproblematically deal with verbs that are of different types cross-linguistically, such as HATE. In a language in which HATE is an agreeing verb, the map simply predicts that contiguous categories should also include agreeing verbs when they border on other categories that include agreeing verbs. Thus, if a language contains agreeing verbs of perception in addition to agreeing verbs of emotion, then it must also have agreeing verbs of cognition in order not to violate the predictions that the map makes.

In addition, the diachronic changes reported by Engberg-Pedersen (1993) and Meir et al. (2007 and later work) can be viewed from a fresh perspective with the use of the semantic map. In these cases, the map makes particular predictions about the possible pathways of change - even if it cannot tell us anything about the direction of change. As a thought experiment to illustrate this point, imagine that all verbs in a particular sign language were bodyanchored at a certain point in time, but, three generations later, a subset of verbs now display agreement properties. On the basis of the semantic map, we predict this subset to include 
verbs that belong to semantic categories connected on the map, e.g. 'effective action', 'contact', 'pursuit', and 'interaction'. The development should only occur along the lines drawn in the map. It would go against prediction to find that, at one point, there were agreeing verbs in the 'effective action' and 'pursuit' classes, but not in the 'contact' class, for instance.

It seems implausible that a sign language ever included only body-anchored verbs; neutral verbs are likely to have formed a part of the language from its inception, too. Unlike body-anchored verbs, neutral verbs are characterized by a place of articulation that does not appear to be iconically motivated. At the same time, it is known that neutral verbs have the potential to be localized, a strategy that can be - and has been - analyzed as another instance of agreement. Perhaps, then, there is a second dimension of change: one from non-localizing to localizing verbs. Again, the expectation would be that such change may only occur along the pathways dictated by the semantic map.

Of course, I do not intend to claim that all verbs eventually become agreeing verbs. It is rather the other way around: if a language has verbs that can overtly agree (with either one or two arguments), they will have developed (i) from either body-anchored verbs or neutral verbs, and (ii) along certain fixed pathways, which can be visualized in a semantic map. Further research is necessary to find out whether this hypothesis bears fruit.

\section{Conclusions}

It can be concluded that each verb type occupies a distinctive and generally contiguous area on the map. Body-anchored verbs predominantly occur along the lower dimension and tend to denote events involving an experiencer. Neutral verbs frequently denote either prototypically transitive or prototypically intransitive meanings, although there are also a handful of verb forms that belong in the 'reflexive/middle' and 'spontaneous' categories connecting these two poles. Agreeing verbs are clustered around the upper left part of the map, which includes categories of verbs that select subjects with a relatively high degree of agentivity. Hybrid forms are consistently found in categories that include more than one verb type.

Clearly, data from other sign languages are needed to test the validity of the map and to determine if more or fewer semantic distinctions are necessary to account for all possible patterns in sign languages. However, one should bear in mind that the more radical the changes are that need to be made, the weaker the claim that transitivity and verb types are governed by common semantic properties.

Yet overall, the results lend credibility to the hypothesis that case-marking systems in spoken languages and the verb type system in sign languages are sensitive to the same underlying semantic factors, underscoring the centrality of these notions in language. ${ }^{6}$ This outcome opens up many opportunities for future research. As discussed in the previous section, an obvious question is whether diachronic change indeed occurs along the pathways indicated in the map. Although diachronic data for sign languages is scarce, the few available sources provide at least some indication that this is the case.

\section{Acknowledgments}

I wish to thank Roland Pfau, Enoch Aboh, Vadim Kimmelman, and Helen Koulidobrova for their inquisitive comments and useful suggestions on different versions of this work, as well

\footnotetext{
${ }^{6}$ Of course, this in no way means that morphosyntactic factors cannot also be at play. Indeed, they most likely must, since verb semantics - although governing both - does not determine case marking or verb type.
} 
as the FEAST audience for their much appreciated feedback. I gratefully acknowledge the support of the Dutch Science Foundation (NWO) under grant no. 360-70-520.

\section{References}

Blanck, Dolly, et al. 2010. "The DGS Corpus project - Development of a corpus based electronic dictionary German Sign Language - German.” Poster session presented at Theoretical Issues in Sign Language Research 10, West Lafayette, IN. https : / / www . signlang.uni-hamburg.de/dgskorpus/index.php/publications.html.

de Quadros, Ronice Müller. 1999. "Phrase structure of Brazilian Sign Language.” PhD thesis, Pontifícia Universidade Católica do Rio Grande do Sul.

de Quadros, Ronice Müller, and Josep Quer. 2008. "Back to back(wards) and moving on: On agreement, auxiliaries and verb classes in sign languages." In Proceedings of the Theoretical Issues in Sign Language Research Conference 9, ed. by Ronice M. de Quadros, 530-551. Petrópolis: Arara Azul.

Dixon, Robert M. W. 1979. “Ergativity”. Language 55:59-138.

Engberg-Pedersen, Elisabeth. 1993. Space in Danish Sign Language. Hamburg: Signum.

Hartmann, Iren, Martin Haspelmath, and Bradley Taylor, eds. 2013. Valency Patterns Leipzig. Leipzig: Max Planck Institute for Evolutionary Anthropology. http://valpal . info/.

Haspelmath, Martin. 1999. "External possession in a European areal perspective." In External possession, ed. by Doris Payne and Immanuel Barshi, 109-135. Amsterdam: John Benjamins.

— .2003. "The geometry of grammatical meaning: Semantic maps and cross-linguistic comparison." In The new psychology of language, ed. by Michael Tomasello, 2:211-243. New York: Lawrence Erlbaum Associates Publishers.

Hengeveld, Kees, and Eva van Lier. 2010. “An implicational map of parts of speech.” Linguistic Discovery 8(1):129-156.

Hopper, Paul J., and Sandra A. Thompson. 1980. “Transitivity in grammar and discourse.” Language 56(2):251-299.

Janis, Wynne D. 1992. “Morphosyntax of the ASL verb phrase.” PhD thesis, State University of New York at Buffalo.

Kemmer, Suzanne. 1993. The middle voice. Amsterdam: John Benjamins.

Levin, Beth. 1993. English verb classes and alternations: A preliminary investigation. Chicago: University of Chicago Press.

Malchukov, Andrej. 2005. "Case pattern splits, verb types and construction competition." In Competition and variation in natural languages. The case for case. Ed. by Mengistu Amberber and Helen de Hoop, 73-117. Oxford: Elsevier.

Meir, Irit. 1998. “Thematic structure and verb agreement in Israeli Sign Language.” PhD thesis, University of Haifa.

— . 2002. "A cross-modality perspective on verb agreement." Natural Language \& Linguistic Theory 20:413-450.

— . 2012. "The evolution of verb classes and verb agreement in sign languages." Theoretical Linguistics 38(1-2):145-152. 
— . 2016. "Grammaticalization is not the full story: A non-grammaticalization account of the emergence of sign languages agreement morphemes". In Quo vadis morphology? MMM10 Online Proceedings, ed. by Jenny Audring, Francesca Masini, and Wendy Sandler, 112-124.

Meir, Irit, Carol Padden, Mark Aronoff, and Wendy Sandler. 2007. “Body as subject”. Journal of Linguistics 43:531-563.

Oomen, Marloes. 2017. "Iconicity in argument structure: Psych-verbs in Sign Language of the Netherlands." Sign Language \& Linguistics 20(1):55-108.

Padden, Carol. 1988. Interaction of morphology and syntax in American Sign Language. New York: Garland.

Sloetjes, Han, and Peter Wittenburg. 2008. “Annotation by category - ELAN and ISO DCR.” In Proceedings of the Sixth International Conference on Language Resources and Evaluation. Marrakech, Morocco: European Language Resources Association.

Tsunoda, Tasaku. 1981. "Split case-marking in verb types and tense/aspect/mood". Linguistics 19:389-438.

\section{Appendix}

See the next page. 


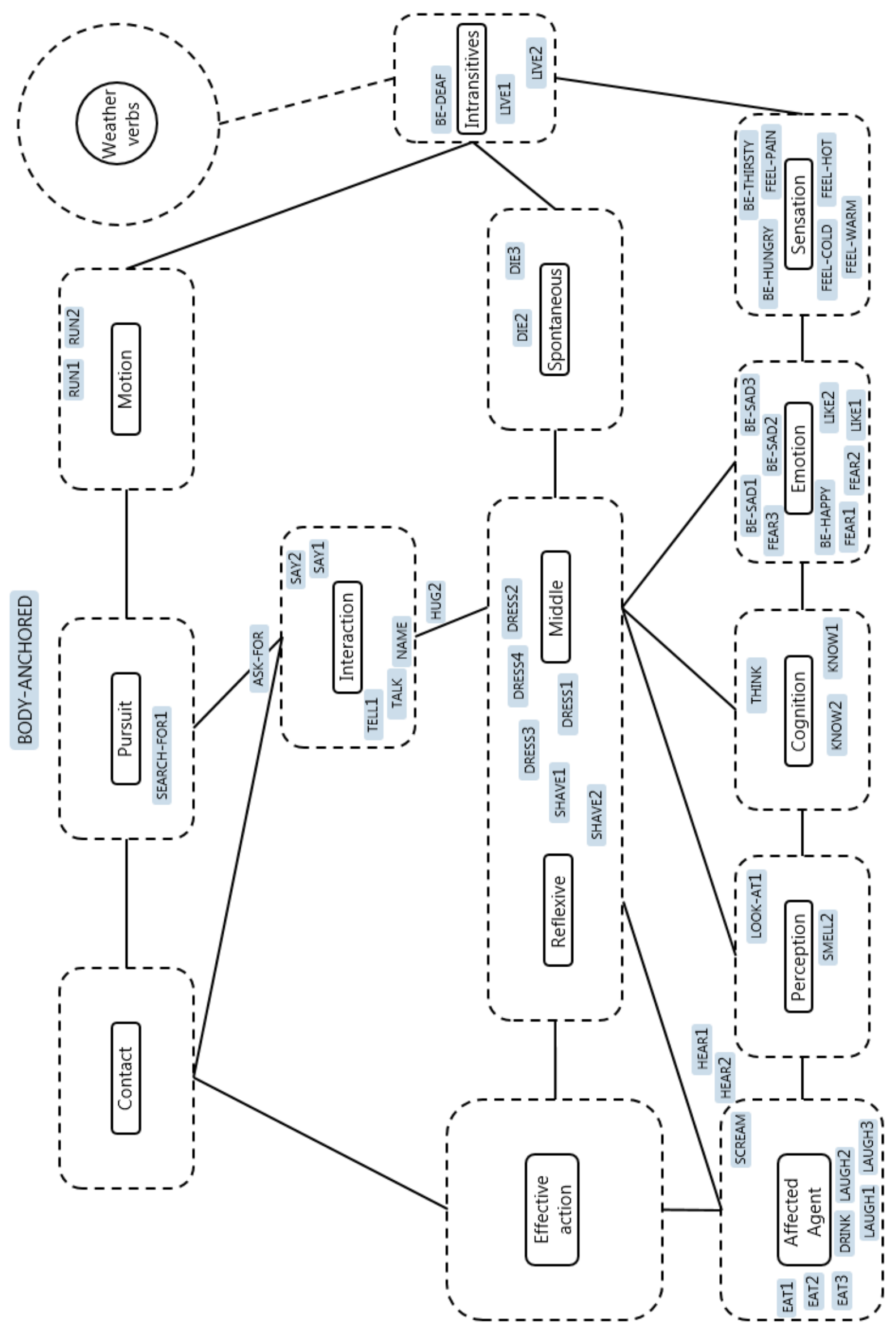

Figure 6: The semantic map for transitivity splits (Malchukov 2005) with DGS body-anchored verbs. 


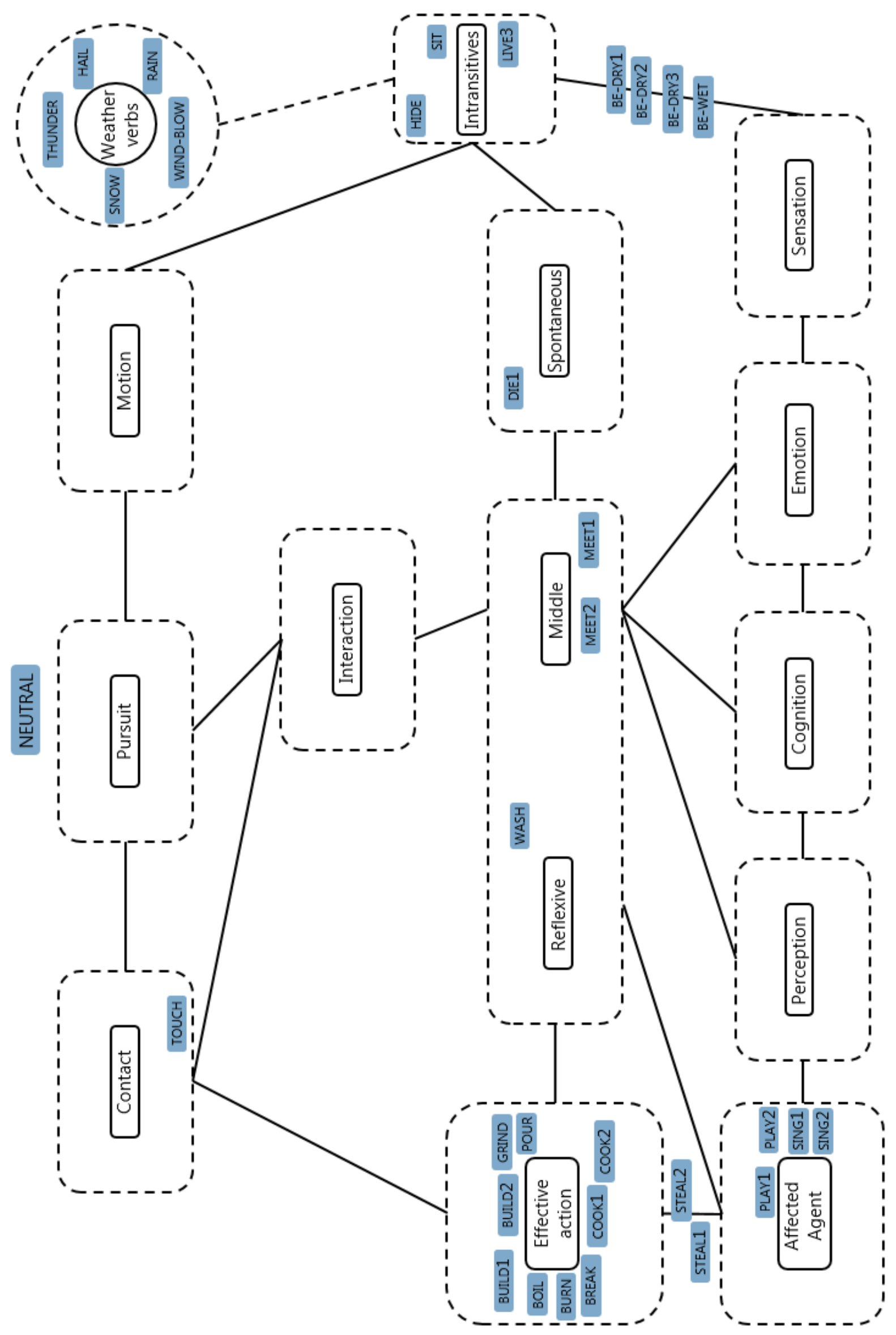

Figure 7: The semantic map for transitivity splits (Malchukov 2005) with DGS neutral verbs. 


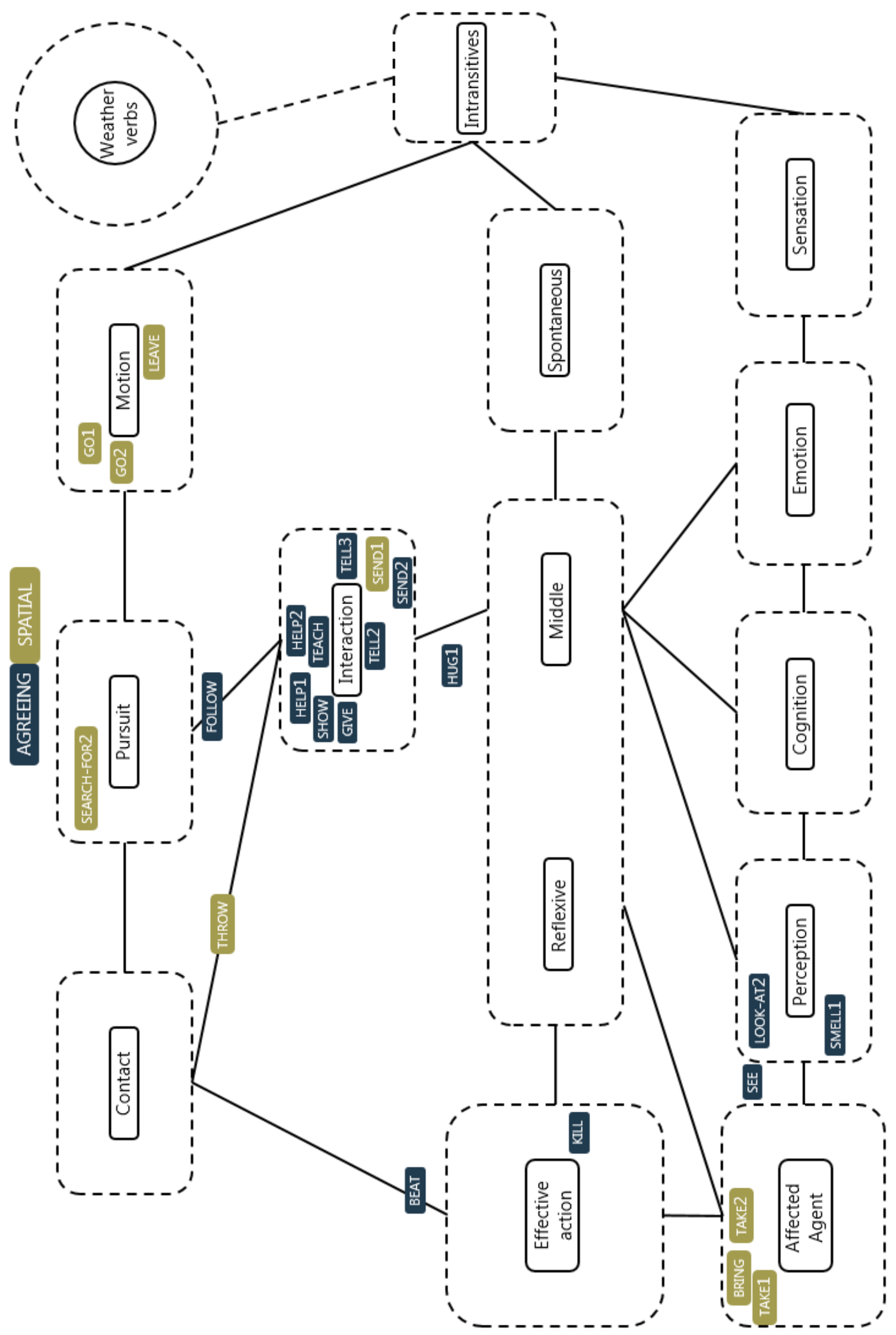

Figure 8: The semantic map for transitivity splits (Malchukov 2005) with DGS regular agreeing verbs (blue) and spatial verbs (green). 